\title{
Extensive mandible torus: bone density study, excision and histological findings
}

\author{
Marcelo Rodrigues Azenha 1*, Giovani Antonio Rodrigues 1, Davi Ariel Nabuo Bepu 1, Alan Grupioni \\ Lourenço 1, Plauto Christopher Aranha Watanabe 1
}

${ }^{1}$ Ribeirão Preto School of Dentistry, University of São Paulo, FORP/USP, Brazil.

${ }^{*}$ Corresponding author: Marcelo Rodrigues Azenha. Ribeirão Preto School of Dentistry - University of São Paulo. Av. do Café, s/n. Zip Code: 14040-904, Ribeirão Preto, São Paulo, SP, Brazil. Phone: +55 (16) 9 9636-6409. E-mail: marceloazenha@usp.br.

Research Ethics Committee Approval (if necessary): We declare that the patient approved the study by signing an informed consent form and the study followed the ethical guidelines established by the Declaration of Helsinki.

Received on: Jul 18, 2021. Accepted on: Jul 27, 2021. Available online: Mon X, 2021.

Exostoses are congenital and benign enlargements of bone tissue occurring in the oral cavity receiving different nomenclatures depending on the area in which they are found. The palatine torus is a bony exostosis that occurs in the midline of the hard palate while the Mandibular Torus (MT), not as common as the palatine, develops on the lingual surface of the mandible. These developmental changes are not considered pathologies for some authors, who classify them as variations of normality. It presents as a bony lobes protuberance usually located in the region of the canine teeth and lower premolars above the mandibular myohyoid line bilaterally [1].

MT has an estimated prevalence as high as $33 \%$ with a variety of genetic and behavioral factors like temporomandibular dysfunction and bite force. MT is a common incidental finding and typical imaging features include a mineralized bone arising from the lingual mandible. Histologically, TM is classified as an exostosis consisting of hyperplastic bone with mature cortical and trabecular elements. Extensive bone growth in the jaw may be associated with poor feeding and phonation [2].

In the images, we observe a sessile base bone mass located bilaterally in the mandible of approximately $20 \times 60 \mathrm{~mm}$ in length classified as MT (Figure 1A to 1B). It was a heteromorphous bone growth with four lobes at the right side of the mandible and three lobes at the left side (Figure $1 \mathrm{~A}$ to 1B). Patient did not complain of pain but reported impaired speech and swallowing, as well as food retention in the torus lobes after meals. The bone density profile of the lesion was also compared to the mandibular 
Extensive mandible torus bone density study excision and histological findings

cortex, thus corroborating the hypothesis of bone exostosis (Figure 1E).

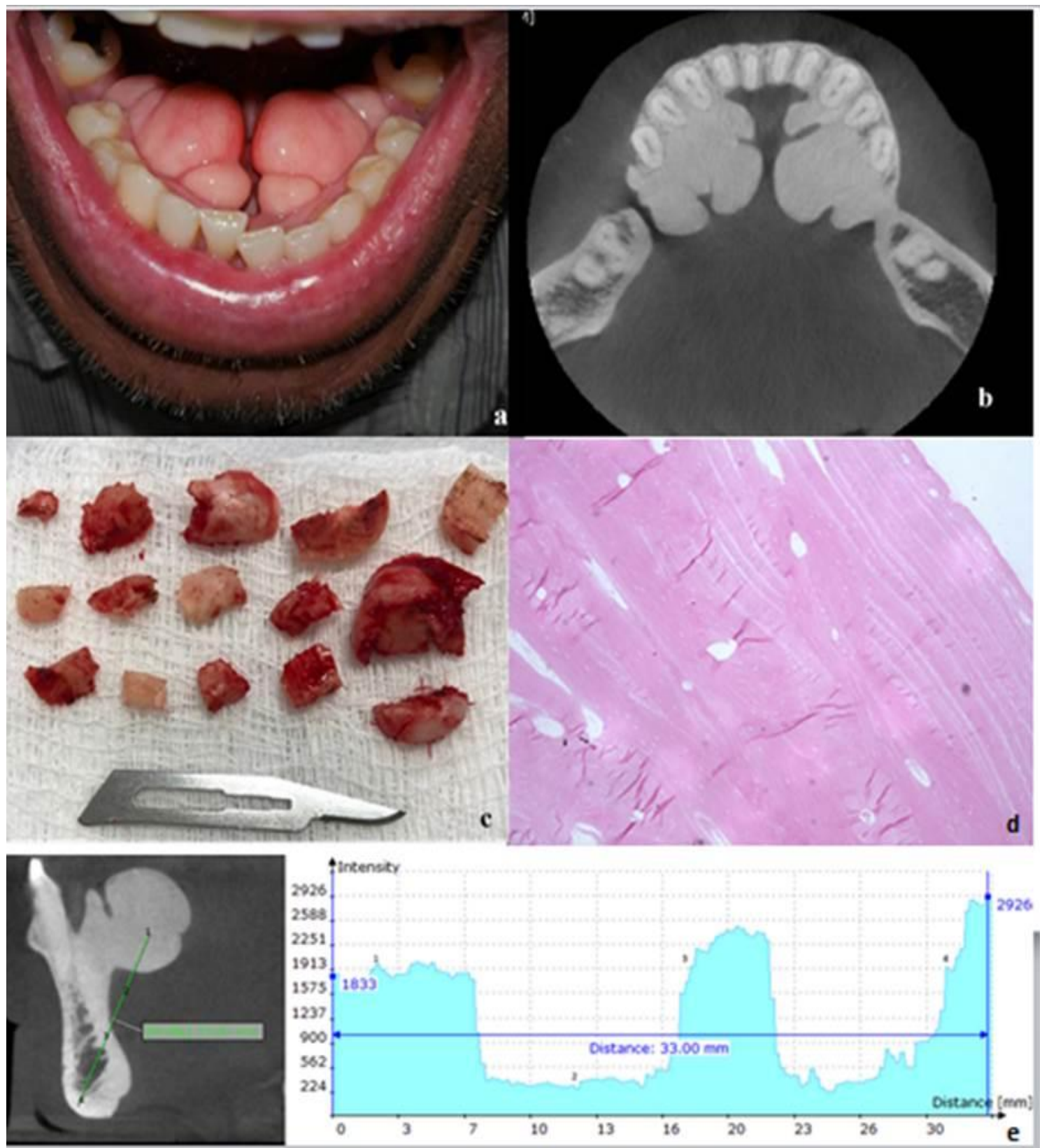

Figure 1. Intraoral view of MT (a); CT of the mandible (b); bone removed after surgical excision (c); histological findings (d); bone density of the MT similar to mandibular bone (e).

Surgical excision of the bone was performed under local anesthesia (Figure 1C) with histological examination revealing the presence of a dense lamellar mass of cortical bone confirming the diagnosis of MT (Figure 1D). The majority of the patients diagnosed with MT has few or no complain and do not require surgical excision. Otherwise, individuals with 
recurrent ulceration and chewing difficulty are serious candidates to have the exostosis removed. In general, the situations that require surgical excision of the MT are routine ulcers caused by solid foods, chewing problems, gland's duct obliteration, food retention and hygiene difficulties, severe tongue dislocation causing breathing difficulty, and in situations where there is a need to use dental prostheses. We report a situation where the patient related the first signs of the MT 20 years ago reporting that there has been a significant increase of the bone in the last years. He complained of sleep disorders, mucosal laceration during feeding, and difficulty of mouth cleaning [3].

Imaging plays an important role in MT identification and computer tomographic exams are commonly employed in our routine and was the choosen exam in this particular case. Using some tools in the computer we were able to determine bone density profile of the exotosis and compare to the mandibular cortex, thus corroborating the hypothesis of MT. Surgical MT removal was efficient to improve patient's quality of life related to feeding, phonation and breathing.

\section{References}

[1] Khan S, Haider Shah SA, Ali F, Rasheed D. Concurrence of torus palatinus, torus mandibularis and buccal exostosis. J Coll Physicians Surg Pakistan. 2016;26:111-113.
[2] Ajay A Madhavan, Robert J McDonald, Felix E Diehn, Carrie M Carr, Jared $\mathrm{T}$ Verdoorn. Giant torus mandibularis causing submandibular duct obstruction and sialadenitis. Neuroradiol Journal 2021, 34(3) 249-252. doi: $10.1177 / 1971400920978431$.

[3] Reichart PA, Neuhaus F, Sookasem M. Prevalence of torus palatinus and torus mandibularis in Germans and Thai. Community Dent Oral Epidemiol. 1988;16(1):61-64. doi: 10.1111/j.16000528.1988.tb00557.x.

Conflict of interest: The author declares no conflicts of interest.

Acknowledgements: None.

Funding: None.

How to cite this article: Azenha MR, Rodrigues GA, Bepu DAN, Lourenço AG, Watanabe PCA. Extensive mandible torus: bone density study, excision and histological findings. Brazilian Journal of Case Reports. 2021 Jul-Sep; 01(3):41-43. 